\title{
Ein neues, kontextualisiertes Maß für Demokratie Konzeptualisierung und Operationalisierung*
}

In der empirischen Demokratieforschung wird mit der Identifikation von Defiziten bzw. der Suche nach einer Varianz in der Demokratiequalität etablierter Demokratien einem neuen Aspekt der Demokratiemessung vermehrt Aufmerksamkeit geschenkt (Diamond/Morlino 2004). Mit diesem Beitrag wird die Idee einer kontextualisierten Demokratiemessung (Abromeit 2004) weiter vorangetrieben, die es ermöglichen soll, auch Unterschiede in der Qualität etablierter Demokratien zu bestimmen. Ich verfolge das Ziel dabei in zwei Stufen: Zunächst soll es mittels eines neu entwickelten kontextualisierten Modells ermöglicht werden, aufgrund der vorgenommen Operationalisierung qualitativ für jedes (demokratische) Land Defizite in der demokratischen Qualität zu identifizieren. Darüber hinaus wird ein quantitativer Index eingeführt, sodass auch ein Vergleich der demokratischen Qualität zwischen Staaten möglich wird.

Inhalt

1. Einleitung

2. Theoretische Grundlagen 211

a) Die Demokratiequalität demokratischer Staaten $\quad 211$

b) Das Konzept von Heidrun Abromeit 213

3. Demokratische Qualität, der Kontext und ihre Beziehung 215

a) Die Logik des kontextualisierten Modells $\quad 215$

b) Die Verbindung von Kontext und Beteiligungsstrukturen 217

4. Operationalisierung und Messung 220

a) Das optimale Mischverhältnis zwischen direkten und repräsentativen Instrumenten 220

b) Das optimale Angebot an Opportunitätsstrukturen 221

c) Die Effektivität der bestehenden Opportunitätsstrukturen 224

$\begin{array}{ll}\text { 5. Fazit } & 227\end{array}$

$\begin{array}{ll}\text { Literatur } & 228\end{array}$

* Ich möchte den Teilnehmern der Sektionstagung „Vergleichende Politikwissenschaft“ im November 2007 für hilfreiche Diskussionsbeiträge zu einer früheren Version dieses Beitrags danken. Des Weiteren danke ich Heidrun Abromeit, Jörg Kemmerzell und einem anonymen Gutachter für wertvolle Hinweise. 


\section{Einleitung}

Nicht zuletzt durch den anhaltenden Siegeszug der Demokratie zum Ende des 20. Jahrhunderts entwickelte sich im Feld des empirischen Demokratievergleichs der Bedarf nach verfeinerten theoretischen Konzepten und Messkonzepten, um der Vielfalt an demokratischen und nicht-demokratischen Herrschaftsformen gerecht zu werden. Die traditionelle Unterscheidung von Demokratien und Nicht-Demokratien reichte nicht mehr aus, weshalb sich seit den 80er-Jahren die qualitative und quantitative Demokratiemessung etablierten. Den seither entwickelten „klassischen“ Demokratiemessungen wie z. B. den Polity-Scores, Freedom House und dem Vanhanen-Index ${ }^{1}$ gelang es, die breiten Übergangszonen zwischen Diktaturen und Demokratien besser zu erfassen. Doch identifizieren fast alle Messungen kaum Varianz in der demokratischen Qualität von etablierten Demokratien. Es ist jedoch unbestritten, dass selbst diese mit Defiziten zu kämpfen haben, die jedoch durch die zumeist zu groben Indikatoren der bestehenden Messungen nicht aufgedeckt werden können. Eine Möglichkeit besteht in einem typologischen Vorgehen, um die Graustufen zwischen den Idealtypen zu beleuchten. Doch werden mit Konzepten wie z. B. der Defekten Demokratie (Merkel 1999, 2003; Croissant/Thiery 2000) vor allem Defizite von Transformationsstaaten in der Konsolidierungsphase erfasst (Merkel u. a. 2003, 2006).

Die Bestimmung der demokratischen Qualität innerhalb der Gruppe der etablierten Demokratien ist erst in jüngerer Zeit in den Fokus der Forschung gerückt. Diamond/Morlino (2004) wählen hierfür den Weg, neben der Qualität der demokratischen Prozesse auch deren Inhalte und Ergebnisse als relevante Elemente der demokratischen Qualität zu berücksichtigen. Auch das Projekt „Democracy Barometer“ geht dafür von einem minimalen Demokratieverständnis $\mathrm{ab}$ und nimmt weitreichende Ausweitungen im Verständnis der Basisdimensionen Gleichheit und Freiheit vor (Bühlmann u. a. 2006, 2007). Doch alternativ sollten zudem auf Basis eines möglichst minimalen Demokratie-Verständnisses Unterschiede in der Demokratiequalität aufgedeckt werden können, um dem Problem, dass erst die Erweiterungen des Demokratieverständnisses zur Identifikation von Defiziten führe, zu entgehen.

In diesem Beitrag stelle ich ein neues Modell mit einem Messindex vor. Es soll auf Basis eines minimalen Demokratieverständnisses als vordringlichste Stoßrichtung das Aufdecken von Varianz in der demokratischen Qualität etablierter Demokratien ermöglichen, darüber hinaus aber auch als allgemeines Demokratiemaß anwendbar sein. Ich schließe an Heidrun Abromeits (2004) konzeptionelle Idee an, zu diesem Zweck den jeweiligen (gesellschaftlichen und institutionellen) Kontext zu berücksichtigen. Das Ziel soll in zwei Stufen erreicht werden: Zunächst erlaubt die vorgenommene Konzeptualisierung und Operationalisierung, qualitativ für jedes (demokratische) Land Defizite in der demokratischen Qualität zu identifizieren. Darüber hinaus wird die Messung in einen quantitativen Demokratie-Index überführt, sodass auch ein Vergleich zwischen Staaten möglich wird.

Ich werde dazu zunächst die bisherigen Überlegungen zur Messung demokratischer Qualität diskutieren und die theoretischen Grundlagen des kontextualisierten Modells darstellen (Abschnitt 2), bevor es in Abschnitt 3 formalisiert eingeführt wird. Dort werden

1 Einen Überblick über die Messungen und den Forschungsstand bieten Schmidt 2000; Lauth/Pickel/ Welzel 2000; Munck/Verkuilen 2002; Lauth 2004. 
auch die Verbindungen von Kontext und Beteiligungsstrukturen genauer diskutiert. In Abschnitt 4 folgt eine erste Operationalisierung der einzelnen Elemente des Modells, die eine qualitative und quantitative Anwendung ermöglichen soll.

\section{Theoretische Grundlagen}

\section{a) Die Demokratiequalität demokratischer Staaten}

Geht es nach Freedom House oder den Polity-Scores, so gibt es kaum Varianz in der demokratischen Qualität innerhalb der Gruppe der „Demokratien“. Betrachtet man entweder die OECD-Mitglieder oder die dauerhaften Demokratien aus Lijpharts Analyse (1999), so fallen lediglich Ausnahmen wie die Türkei und Mexiko (OECD) oder neuerdings auch markanter einige Demokratien aus Lijpharts Sample wie z. B. Kolumbien und Venezuela mit abweichenden, tendenziell weniger demokratischen Werten auf. Die klassischen „westlichen“ Demokratien erscheinen dagegen fast alle als perfekt. ${ }^{2}$

Doch stellt sich spätestens seit der Messung von Vanhanen (1997) die Frage nach einer möglichen Varianz auch innerhalb dieser Gruppe. Laut dessen Demokratie-Index sind z. B. die Schweiz und die USA halb so demokratisch wie Italien (Vanhanen 1997: 86). Nun mag man schnell übereinkommen und Vanhanens Operationalisierung seiner Dimensionen als unzureichend kritisieren (Lauth 2004: 247 f.; Schmidt 2000: 400 ff.). Doch sind die Ergebnisse zumindest interessante Hinweise auf unterschiedliche Funktionsweisen, die eventuell zum Auffinden von Defiziten führen können.

Alle drei Messungen basieren letztlich auf Dahls Demokratievorstellung der „Polyarchie“ (1971), nach der Freiheit und Gleichheit in Form von Partizipation und Wettbewerb umzusetzen sind. Dabei geht Freedom House mit der Dimension der bürgerlichen Freiheiten über Dahl und die beiden anderen Messungen hinaus, deren Fokus auf den politischen Rechten verbleibt. Als mögliche Lösung auf der Suche nach Varianz wird von vielen Autoren daher ein anspruchsvolleres, alles andere als minimalistisches Demokratieverständnis herangezogen, das dann anhand der höheren Hürden die Identifikation von Defiziten ermöglicht.

Insbesondere für die schon bei Freedom House und den Polity-Scores gefundenen abweichenden Fälle in der Grauzone zwischen Demokratie und Autokratie wurde mit der Defekten Demokratie (Merkel 1999, 2003; Croissant/Thiery 2000) ein empirisch anwendbares Konzept entwickelt, das über ein minimalistisches Verständnis einer reinen Wahldemokratie hinausgeht, um Funktionsdefizite zu finden. Das Konzept der „embedded democracy“ reicht

2 Allein Griechenland und Japan erhalten bei Freedom House bei den Bürgerrechten lediglich einen Wert von 2, bei den Polity Scores erhält nur Frankreich aufgrund des semi-präsidentiellen Arrangements einen Demokratiewert von 9 anstelle des Maximalwerts von 10. Die aktuellen Werte finden sich unter: http:// www.freedomhouse.org/template.cfm?page=363\&year=2007 und http://www.cidcm.umd.edu/polity/ data/. Auch Lauths (2008) Neuer Index der Demokratie (NID) erbringt kaum ein Mehr an Varianz, obwohl er neben den Polity-Scores und Freedom House auch das Ausmaß an Rechtsstaatlichkeit und die Staatlichkeit in Form eines funktionierenden Gewaltenmonopols als politische Stabilität (beides aus der Governance-Datenbank der Weltbank) berücksichtigt (http://www.fernuni-hagen.de/polwiss/institut/projekte/39936_lg5.html). 
in zweierlei Hinsicht über das Dahl'sche Verständnis hinaus. Es werden mit den ökonomischen Voraussetzungen und sozialer Gerechtigkeit zwei Ermöglichungsbedingungen definiert, die zunächst kein Demokratiemerkmal an sich sind, jedoch Schutz gegen innere und äußere Schocks bieten und damit zur Systemstabilität beitragen (Merkel 2003: 47). Noch wichtiger ist die Erweiterung des eigentlichen Demokratieverständnisses um eine Kontrolldimension, die in Form zweier Teilregime, nämlich der horizontalen Verantwortlichkeit und der effektiven Regierungsgewalt, Einzug hält. Diese Erweiterung über die Basisdimensionen Freiheit und Gleichheit ${ }^{3}$ hinaus ermöglicht die Identifikation von spezifischen Defiziten und empirischen Subtypen.

Eine andere Möglichkeit mit explizitem Fokus auf die etablierten Demokratien stellt Beethams (1994) democratic audit dar, dessen wissenschaftliches Hauptziel ${ }^{4}$ es ist, die demokratische Qualität des jeweils eigenen politischen Systems zu bestimmen. Auch er erweitert dafür sein Demokratieverständnis, indem er als eine von vier Unterdimensionen neben freien Wahlen, Bürgerrechten, politischen Rechten und verantwortlichem Regieren die ,democratic society“ (1994: 28) identifiziert. Diese ist notwendig, um die vertikale Kontrolle der politischen Inhalte oder der Repräsentanten zu gewährleisten. Eine funktionierende Zivilgesellschaft als Demokratie-Anforderung geht natürlich über ein minimales Verständnis weit hinaus. Die auf das jeweils eigene System auszurichtenden Analysekriterien machen zudem einen Vergleich sehr schwer.

Auch Diamond/Morlino (2004: 21) gehen auf ihrer Suche nach Kriterien zur Beurteilung von demokratischer Qualität zunächst von einem minimalen Verständnis aus, indem sie auf allgemeines Wahlrecht, freie Wahlen, Parteienwettbewerb und Informationsfreiheit abheben. Auf dieser Basis stellt sich für die Autoren die Frage, wie gut mittels dieser Institutionalisierungen die Ziele einer idealen Demokratie (Freiheit, vertikale Kontrolle und Gleichheit) erreicht werden. Darüber hinaus sind aber auch breitere Standards des ,good governance“ wie Transparenz und verantwortliches Regieren demokratierelevant. Als Erste versuchen sie neben dem Demokratieverständnis auch ein dreidimensionales Verständnis von Qualität zu definieren. Die prozedurale Qualität beurteilt die politischen Prozesse danach, inwieweit diese unter der Kontrolle der Bürger stehen. Die Qualität des Inhalts bezieht sich auf die Funktionsweise in der Umsetzung der Freiheits- und Gleichheitsrechte. Die Qualität der Ergebnisse wird daran gemessen, inwieweit die Bürger mit denselben zufrieden sind (Diamond/Morlino 2004: 22).

Im Projekt des „Democracy Barometer"5 starten die Autoren ebenfalls bei einer Minimaldefinition von Demokratie, nach der Freiheit und Gleichheit in ihrer minimalen Bedeutung gesichert werden sollen (Bühlmann u. a. 2006: 7). Sie erweitern jedoch ihr Verständnis und fordern zudem, dass in Demokratien „Freiheit und Gleichheit für freie und gleiche politische Selbst- und Mitbestimmung gesichert und optimiert werden“ (Bühlmann u. a.

3 Jene werden mittels der Teilregime ,politische Partizipationsrechte“ und „,bürgerliche Freiheitsrechte“ bzw. des zentral verorteten „Wahlregimes“ erfasst (vgl. Merkel 2003: 55).

4 Darüber hinaus hat der audit den politischen Anspruch, durch die Beteiligung der Bevölkerung an seiner Erstellung den reflexiven Umgang mit der eigenen Demokratie zu üben und somit die Demokratiequalität zu erhöhen.

5 Vgl. http://www.nccr-democracy.uzh.ch/nccr/knowledge_transfer/ip14 (Stand: 02.06.08). 
2006: 10). Damit tritt an die Stelle einer Sicherung von Minimalstandards die Forderung nach einer Maximierung der Demokratiequalität. Der Qualitätsbegriff wird in Analogie zur Produktqualität dreidimensional gedeutet. Die Funktionalität in Form der Demokratie-Garantien findet sich in der jeweiligen institutionellen Ausgestaltung. Zweites Qualitätskriterium ist die Effektivität, nämlich in welchem Ausmaß Freiheit und Gleichheit tatsächlich umgesetzt werden. Zuletzt werden in Analogie zur Benutzerfreundlichkeit die DemokratiePolitiken herangezogen, die eine Verbesserung der ersten beiden Qualitätskriterien zum Ziel haben (Bühlmann u. a. 2006: 20).

Den skizzierten Konzepten liegen unterschiedlich gewichtete Erweiterungen eines minimalen Demokratieverständnisses zugrunde, die in der Konkretisierung auf der nächsten Stufe des „,concept trees“ (Munck/Verkuilen 2002) zur Identifikation teilweise unterschiedlicher Mess-Dimensionen führen. Das birgt die Gefahr, dass je nach konzeptioneller Erweiterung die empirischen Ergebnisse maßgeblich beeinflusst werden. Diese können somit angreifbar werden, da sie auf einem über einen minimalen Grundkanon hinausreichenden Demokratieverständnis beruhen. Daher schlage ich den alternativen Weg vor, ein Konzept zu entwickeln, das auf Basis eines möglichst minimalen Demokratieverständnisses in der Lage ist, Varianzen zu entdecken. Der Aspekt der Qualität an sich ist von besonderem Interesse, denn es ist schon intuitiv klar, dass nicht nur das Vorhandensein von institutionellen und prozeduralen Regelungen, sondern deren Effektivität in der Funktionsweise zu berücksichtigen ist. Dieser Aspekt der Effektivität als zentrales Qualitätsmerkmal soll in das nun vorzustellende Konzept von Heidrun Abromeit (2004) integriert werden.

\section{b) Das Konzept von Heidrun Abromeit}

Abromeit (2004) geht einen anderen Weg als die funktionalistischen Demokratiemodelle der „embedded democracy“ oder des „Democracy-Barometer“, um Unterschiede in der demokratischen Qualität etablierter westlicher Demokratien festzustellen. Sie kritisiert an den klassischen Messverfahren (Vanhanen, Freedom House, Polity-Scores) ein grundlegendes Problem, nämlich die Orientierung an der Institutionalisierungsform des „Realmodells“ westliche liberale Demokratie. Das verursache einen institutionellen Fehlschluss, indem allein eine Institutionalisierungsform als ideale Vergleichsfolie herangezogen wird (2004: 76). Des Weiteren wurde bislang der gesellschaftliche und institutionelle Kontext der jeweiligen Systeme nicht berücksichtigt, die sehr wohl Einfluss auf die demokratische Qualität nehmen können.

Abromeit argumentiert, dass es zur Beseitigung der Defizite eines universellen, möglichst minimalen und vor allem nicht an bestimmte Institutionalisierungsformen gebundenen Demokratiebegriffs bedürfe. Sie verwendet eine Demokratiedefinition, in der die individuelle Selbstbestimmung in den Vordergrund gestellt ist. „Demokratie ist die Verlängerung der individuellen Selbstbestimmung in den Bereich kollektiver Entscheidungen hinein; sie konkretisiert sich in der Beteiligung der Individuen an den Entscheidungen, von denen sie betroffen, denen sie unterworfen sind.“ (Abromeit 2004: 78)

Dieter Fuchs übt Kritik an dieser Definition, da die von Abromeit formulierte „Rückbesinnung auf den Kern der Demokratie“ mit ihrer Definition nicht erreicht werde (2004: 97). 
Die Abromeit'sche Demokratiedefinition kann jedoch nicht prinzipiell abgelehnt werden, da eine am Individuum orientierte Zugangsweise zur Definition von Demokratie auf einem im Liberalismus verwurzelten Demokratieverständnis und somit auf einer vertragstheoretischen Denkweise basiert (Abromeit 2002: 166). Sie befindet sich damit in der Tradition von Locke und Mill, bei denen das Prinzip der individuellen, sogenannten negativen Freiheit (Berlin 1995: 201 ff.) auch konstitutiv für ein demokratisches Staatswesen ist. Aber auch das Prinzip der positiven Freiheit (Berlin 1995: 211 ff.) ist essenziell für eine an das Individuum gebundene Demokratiedefinition, denn dabei geht es um den Wunsch des Individuums, sein eigener Herr zu sein, also den Wunsch nach politischer Selbstbestimmung in einer demokratischen Regierungsform. ${ }^{6}$

Die Definition von Abromeit führt zu einer Konzentration auf den partizipatorischen Aspekt von Demokratie, sie lässt jedoch offen, ob dieser dann mittels direktdemokratischer oder repräsentativer Instrumente umzusetzen ist. So kann Partizipation der Bürger für Abromeit (2004: 78) explizit mittels der unterschiedlichen Verfahrenstypen Mitgestaltung, Widerspruch und Autorisierung von Entscheidungsträgern verwirklicht werden. Das bedeutet jedoch keine Festlegung auf das Modell der partizipatorischen Demokratie (so Fuchs 2004: 98).

An dieser Stelle kommt nun der Kontext ins Spiel, da von ihm abhängt, welches die geeigneten Institutionalisierungsformen sind, um das Ziel der individuellen Selbstbestimmung in Form einer Beteiligung am kollektiven Entscheidungsprozess zu erreichen. Die Relevanz des Kontextes soll anhand der ersten gesellschaftlichen Kontextvariable deutlich gemacht werden: der Heterogenität einer Gesellschaft. Diese beeinflusst direkt die Wirkung einzelner institutioneller Regelungen für die Beteiligung. So erweist sich z. B. ein Mehrheitswahlrecht in Gesellschaften mit Minderheiten dann als nicht verträglich mit dem Ziel der Beteiligung, wenn sie nicht besonders groß oder regional konzentriert sind. Da die Mitglieder solcher Minderheiten dauerhaft keine Chance zur Repräsentation und damit zur Einspeisung ihrer Präferenzen in den politischen Prozess haben, ergibt sich ein demokratisches Defizit. Als zweite Variable des gesellschaftlichen Kontextes werden die sogenannten Dominanzstrukturen hinzugezogen, die Bindungsphänomene und Abhängigkeitsverhältnisse in einer Gesellschaft berücksichtigen (Abromeit 2004: 86). Aber auch der institutionelle Kontext ist wichtig, da sowohl die Komplexität (dritte Variable) als auch der Grad an Formalisierung oder Informalisierung des Entscheidungssystems (vierte Variable) die Notwendigkeit und Wirkungsweise von spezifischen Partizipationsmöglichkeiten beeinflussen. So sind z. B. die Anforderungen an die Beteiligungsmöglichkeiten in einem Mehrebenensystem anders und umfangreicher als in einem einfachen System.

6 Die Folgerung von Fuchs, dass der „Kern“ der Demokratie sich über die Ursprungsbedeutung allein auf eine kollektive Interpretation des demos-Begriffs reduzieren lasse (Fuchs 2004: 98), weist lediglich in eine entgegengesetzte Interpretationslinie der Demokratietheorie, die an die aristotelische Demokratietradition anknüpft. Die Festlegung auf das Modell der repräsentativen Demokratie und damit das Konzept Dahls stellt nur eine von mehreren denkbaren Konzeptualisierungen dar, die sich aus einer solchen Definition ergeben können. 
Fuchs (2004: 102) bleibt wie viele Befürworter des Dahl'schen Konzepts ${ }^{7}$ direktdemokratischen Elementen gegenüber prinzipiell skeptisch. Doch bedeutet die Berücksichtigung direktdemokratischer Elemente keine Ex-ante-Festlegung auf eine partizipatorische Demokratie als Idealbild für alle Staaten. Vielmehr will Abromeit den jeweiligen Kontext ja gerade deswegen berücksichtigen, um bestimmen zu können, wie hoch der Anteil an direktdemokratischen Elementen in einer gegebenen Demokratie jeweils sein sollte (Abromeit 2004: 83). Durch die explizite Einbindung des (gesellschaftlichen und institutionellen) Kontextes wird es möglich, für jedes Land einen optimalen Bedarf an unterschiedlichen direkten oder repräsentativen Beteiligungsinstrumenten zu definieren. Dieses zentrale Anliegen von Abromeit stellt einen Fortschritt gegenüber den bisherigen Messkonzepten dar.

\section{Demokratische Qualität, der Kontext und ihre Beziehung}

\section{a) Die Logik des kontextualisierten Modells}

Grundlegend für die weitere Ausdifferenzierung des kontextualisierten Modells ist zunächst eine knappe, aber wichtige und folgenreiche Erweiterung der Abromeit'schen Demokratiedefinition. Diese geht von der Dimension der Freiheit im demokratischen Herrschaftsprozess aus und berücksichtigt insofern die Gleichheit, als allen Betroffenen Beteiligungsmöglichkeiten zu eröffnen sind. Die Dimension der Gleichheit wird im Folgenden durch die Erweiterung auf die ,effektive Beteiligung“ stärker betont, was eine Chancengleichheit in der Durchsetzung der jeweiligen individuellen Präferenzen impliziert. Effektive Beteiligung heißt, dass es den Individuen nicht nur möglich ist, am Prozess teilzunehmen und die eigenen Interessen einzuspeisen, sondern dass damit auch die Chance auf eine Beeinflussung des Entscheidungsergebnisses gegeben ist. Dieses Verständnis ist zunächst minimal, weil es von einer formalen, rechtlichen Gleichheit aller Individuen ausgeht, wie es beim britischen Liberalismus ebenfalls der Fall ist. Es ist insofern aber nicht mehr minimal, als Effektivität nicht nur formale Garantien, sondern auch reale Umsetzung verlangt. Ich schließe damit an Böckenförde (1991) an, der die Gewährleistung gleicher Chancen für politische Machtgewinnung fordert. Damit geht jedoch nicht die Forderung nach einer substanziellen oder materiellen Gleichheit im Sinne einer sozialen Demokratie einher (Meyer 2005). Mit der Effektivität der Beteiligung wird gleichzeitig auch das zentrale Qualitätskriterium definiert, das es ermöglichen soll, die Funktionsweise institutioneller Regelungen empirisch zu überprüfen und Varianzen zwischen Ländern zu finden.

Wie von Abromeit betont, werden kollektive Entscheidungen innerhalb eines gegebenen institutionellen und gesellschaftlichen Kontextes getroffen. In unterschiedlichen Kontexten verlangt die Realisierung demokratischer Selbstbestimmung nach unterschiedlichen Instrumenten zur Sicherung demokratischer Qualität. Das bedeutet, dass ein bestimmtes Set an Beteiligungsstrukturen nicht in allen Gesellschaften zur gleichen demokratischen Qualität

7 Zwar abstrahiert auch Dahl selbst von spezifischen Institutionalisierungen, indem sein Konzept auf einem (hier prozedural definierten) Idealtypus aufbaut (Dahl 2000: 35 ff.), bislang erfolgte jedoch noch keine explizite Konzeptualisierung der prozeduralen Kriterien, sondern es kam lediglich zu eher ad hoc durchgeführten Operationalisierungen (Munck/Verkuilen 2002). 
führt. Effektive Beteiligung zur Sicherung demokratischer Qualität ist möglich a) durch die Mitgestaltung der Betroffenen, b) durch Widerspruchsrechte und c) durch die Autorisierung vikarischer Entscheidungsträger (Abromeit 2004: 78). Damit kann das Instrumentarium sowohl direktdemokratische ( $\mathrm{a}$ und b) als auch repräsentative Elemente (b und c) umfassen.

Zur Konstruktion des Modells löse ich mich im Folgenden von Abromeits Operationalisierungsvorschlägen, in denen drei verschiedene Beteiligungsvariablen als Demokratievariablen definiert (2004: 82 ff.) werden. Ich greife vielmehr auf die ausgeführte Aufteilung der effektiven Beteiligung in die beiden grundlegenden Elemente (direktdemokratisch und repräsentativ) zurück. Nach der Logik des Modells determiniert zunächst der gesellschaftliche und institutionelle Kontext den Bedarf an einer spezifischen Zusammenstellung von optimalen Opportunitätsstrukturen. Er ist anschließend den existierenden institutionellen Arrangements an Beteiligungsstrukturen gegenüberzustellen. Doch reicht die Existenz von Partizipationsmöglichkeiten nicht aus, vielmehr müssen sie auch wirksam hinsichtlich der individuellen Selbstbestimmung sein, es ist also deren Effektivität zu ermitteln.

Das Modell eignet sich somit zunächst für ein qualitatives Vorgehen. Ziel ist es, für ein bestimmtes Land gezielt Defizite in der demokratischen Qualität zu identifizieren. Damit ähnelt der Anspruch durchaus Beethams democratic audit (1994). Doch soll darüber hinaus zum Zweck der Vergleichbarkeit von Ländern auch eine Quantifizierung erfolgen. Zur Berechnung des kontextualisierten Maßes an Demokratie schlage ich den folgenden Index DI vor:

$$
\begin{aligned}
& \text { (1) } \mathrm{DI}=(1-\mathrm{k}) \sum_{\mathrm{r}=1}^{\mathrm{n}} \alpha_{\mathrm{r}}\left(\mathrm{e}_{\mathrm{r}} \cdot \frac{\mathrm{osa}_{\mathrm{r}}}{\mathrm{OSO}_{\mathrm{r}}}\right)+\mathrm{k} \sum_{\mathrm{d}=1}^{\mathrm{m}} \alpha_{\mathrm{d}}\left(\mathrm{e}_{\mathrm{d}} \cdot \frac{\mathrm{osa}_{\mathrm{d}}}{\mathrm{OSO}_{\mathrm{d}}}\right) \\
& \text { mit } 0 \leq \mathrm{k} \leq 1,0 \leq \mathrm{e}_{\mathrm{r}} \leq 1, \sum_{\mathrm{r}=1}^{\mathrm{n}} \alpha_{\mathrm{r}}=1,0 \leq \mathrm{e}_{\mathrm{d}} \leq 1, \sum_{\mathrm{d}=1}^{\mathrm{m}} \alpha_{\mathrm{d}}=1
\end{aligned}
$$

Dabei stellt k den optimalen Anteil an direktdemokratischen Instrumenten zur Festlegung des „Mischungsverhältnisses“ (Abromeit 2004: 83) dar, sodass (1 - k) den Anteil an repräsentativen Instrumenten angibt. Die Elemente oso bzw. oso $_{d}$ sind die jeweiligen vom Kontext determinierten einzelnen Beteiligungsmöglichkeiten, in der Summe also das optimale Angebot an repräsentativen bzw. direktdemokratischen Opportunitätsstrukturen (der Bedarf). Die einzelnen Elemente sind dabei noch kontextabhängig zu gewichten $\left(\alpha_{\mathrm{r}} \mathrm{bzw}\right.$. $\left.\alpha_{d}\right)$. Als Beispiel seien parlamentarische Systeme mit direkt gewähltem Präsidenten ohne große Machtressourcen angeführt. Hier sind aufgrund des Einflusses auf die kollektiven Entscheidungen die Wahlen zur Volksvertretung sicherlich höher zu gewichten als die des Präsidenten. In osa bzw. osa $_{d}$ wird jeweils überprüft, ob das tatsächliche Angebot an repräsentativen bzw. direktdemokratischen Opportunitätsstrukturen dem Bedarf entspricht. Die Größen $e_{r}$ bzw. $e_{d}$ stellen schließlich die Effektivität der jeweiligen bestehenden repräsentativen bzw. direktdemokratischen Elemente dar. Durch die Standardisierung aller Werte zwischen 0 und 1 kann der Index DI einen Wert zwischen 0 und 1 annehmen und lässt damit einerseits einen Vergleich der demokratischen Qualität eines Landes mit dem optimalen Zustand (DI = 1) und andererseits auch einen Vergleich zwischen Ländern zu. 


\section{b) Die Verbindung von Kontext und Beteiligungsstrukturen}

Um den Bedarf an Opportunitätsstrukturen - also das optimale Angebot - bestimmen zu können, ist es notwendig, theoretisch begründete Annahmen über die Beziehung zwischen den Kontextvariablen und dem sich ergebenden Bedarf zu formulieren (Abromeit 2004: 85 ff.). Gesellschaftliche Kontextvariablen sind die Heterogenität der Gesellschaft und Dominanzstrukturen, institutionelle Variablen die Komplexität des Entscheidungssystems und der Grad der Formalisierung bzw. Informalisierung des Entscheidungsprozesses. ${ }^{8}$

Von den vier Kontextvariablen ist allein das Ausmaß an Dominanzstrukturen ${ }^{9}$ nicht mit einer konkreten Nachfrage nach bestimmten Opportunitätsstrukturen verbunden. Vielmehr können sie die Effektivität der unterschiedlichen Beteiligungsinstrumente reduzieren. Das gilt sowohl für repräsentative wie auch direktdemokratische Elemente. Denn die gesellschaftliche Dominanz von z. B. Parteien kann dazu führen, die eigentlichen Präferenzen der an sie gebundenen Individuen zu überlagern. So können auch die Ergebnisse von Referenden durch Parteipositionen determiniert sein und somit das Prinzip der Selbstbestimmung unterminieren.

Die folgende Abbildung 1 veranschaulicht die postulierten Beziehungen zwischen den anderen drei Kontextvariablen und dem Bedarf an Beteiligungsstrukturen.

\section{Abbildung 1}

Der Zusammenhang von Kontextvariablen und Beteiligungsarten

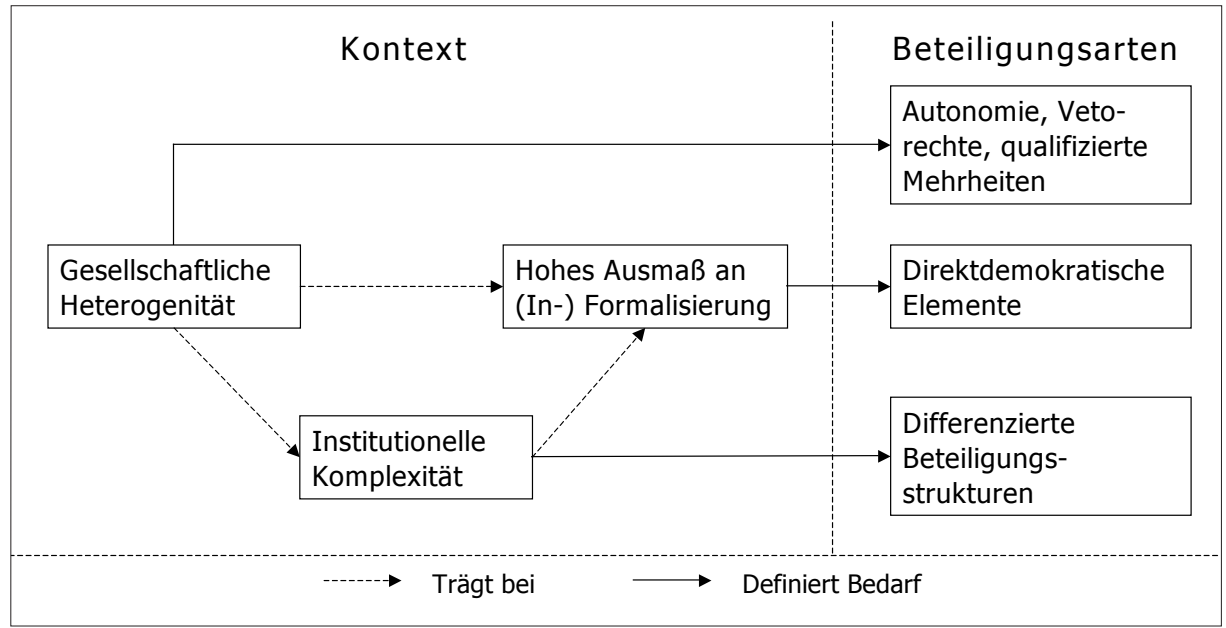

8 Politische Institutionen werden in diesem Zusammenhang als all jene formalen und informellen Regelungen verstanden, die dauerhaft den politischen Prozess strukturieren.

9 Gesellschaftliche Dominanz liegt dann vor, wenn es einer Elite gelingt, den Präferenzbildungsprozess der Individuen zu kontrollieren oder zumindest zu beeinflussen (Abromeit/Stoiber 2006: $153 \mathrm{ff}$.). 
Abromeits (2004: 84) zweite gesellschaftliche Kontextvariable ist die gesellschaftliche Heterogenität. Sie argumentiert, dass eine starke Segmentierung der Gesellschaft und die damit in der Regel verbundenen intensiven Präferenzen zu hohen Frustrationskosten durch wiederholtes Überstimmtwerden führen. Um den gesellschaftlichen Frieden nicht zu gefährden, seien daher Beteiligungsformen zu wählen, die es den Segmenten oder Minderheiten ermöglichen, ihre Präferenzen effektiv einzubringen.

Der Status von Minderheiten und die Begründung besonderer Gruppenrechte werden aus unterschiedlichen theoretischen Perspektiven diskutiert. So fordert Taylor (1993) aus kommunitaristischer Sicht aufgrund des Vorrangs der Sicherung kollektiver Identitäten gegenüber individuellen Freiheiten die Anerkennung ihrer Unterschiede und somit kollektive Rechte für Minderheiten. Anders als diese Argumentation ist Kymlicka (1995) direkt anschlussfähig an das gewählte individualistisch-liberale Demokratieverständnis. Er zeigt, dass individuelle Freiheit vereinbar ist mit der Gewährung von Gruppenrechten für Minderheiten, die die Mehrheitsmacht der Gesamtgesellschaft („externer Druck“) einschränken. Er argumentiert insbesondere mit Gleichheitsgrundsätzen wie der Anerkennung der individuellen Unterschiedlichkeit (Kymlicka 1995: 108). In der Realität unterscheidet er drei Formen gruppenbasierter Minderheitenrechte: Minderheitenrepräsentation, Selbstregierungsrechte und polyethnische Rechte (Kymlicka 1995: 26 ff.). Letztere werden nicht weiter adressiert, da sie vor allem auf die Gewährung von Bürgerrechten für Einwanderer und religiöse Minderheiten abzielen, die den politischen Prozess aber nicht direkt betreffen. Selbstregierungsrechte wie z. B. das Zugeständnis autonomer Regelungsinhalte oder gar regionale Eigenständigkeit sind vor allem bei einer räumlichen Konzentration von Minderheiten möglich. Dagegen bewirken spezielle Repräsentationsprivilegien auf nationaler Ebene oder niedrige Repräsentationshürden die Einspeisung der Minderheiteninteressen in den gesamtgesellschaftlichen Politikprozess.

An diesem Punkt schließt Lijphart an, indem er die Problematik von ethnischen Minderheiten auf segmentierte Gesellschaften im Allgemeinen ausweitet. Er argumentiert, dass in politischen Systemen, die durch ihre Gründung schon von vornherein als segmentiert festgelegt waren, explizit oder implizit qualifizierte Mehrheiten notwendig seien. Verhältniswahl und große Koalitionen sind gegebenenfalls mit der Gewährung von Autonomierechten oder dem Minderheitenveto, der stärksten Institutionalisierungsform, zu verbinden (Lijphart 1995: 278). So sind anstelle der Mehrheitsregel für Entscheidungen auf zentralstaatlicher Ebene qualifizierte Mehrheiten anzuwenden, wenn es um den grundgesellschaftlichen Konsens geht. Sind Entscheidungsgegenstände für die betroffenen Gruppen äußerst sensibel und von essenzieller Bedeutung für deren Identität, sind dagegen Bereiche autonomer Entscheidungen oder alternativ Vetorechte einzuräumen.

Gesellschaftliche Heterogenität kann auch dazu beitragen, dass ein komplexes Entscheidungssystem entsteht. In einem solchen Kontext werden differenzierte Beteiligungsstrukturen notwendig. Darunter fällt eine für den jeweiligen Kontext sensible Möglichkeit, die Beteiligung der Betroffenen zu ermöglichen. Das trifft für Mehrebenensysteme zu, Systeme vermehrter checks and balances oder sektoral differenzierte Entscheidungssysteme. Effektive Beteiligung muss auf allen für die Betroffenen relevanten Ebenen möglich sein. Ziel ist 
es, dass alle Entscheidungsträger in komplexen Systemen tatsächlich demokratisch legitimiert sind oder die Entscheidungen durch die Betroffenen kontestierbar sind.

Ein hoher Grad an (In-)Formalisierung kann sich unter Umständen aufgrund hoher gesellschaftlicher Heterogenität aber vor allem aus einem komplexen Entscheidungssystem ergeben. Insbesondere in komplexen Systemen herrscht oftmals eine Überformalisierung der Entscheidungsstrukturen. Das kann dazu führen, dass die politische Elite sich immer weiter vom Demos entfernt. Das Prinzip der Verantwortlichkeit (accountability), das dafür sorgt, dass die Präferenzen der Individuen das Handeln der gewählten Vertreter bestimmen, wird so unterlaufen. Um die getroffenen Entscheidungen wieder an den Willen der Betroffenen zurückzubinden, werden daher direktdemokratische Instrumente notwendig. Auf der anderen Seite entsteht durch die Notwendigkeit zu verhandeln häufig eine Informalisierung der Politik verbunden mit einer Auswanderung aus den Institutionen (von Blumenthal 2003), um in heterogenen Gesellschaften einen zumindest minimalen Konsens zu gewährleisten, oder um in komplexen Systemen überhaupt Entscheidungen treffen zu können. Als Beispiel hierfür sei der kooperative Föderalismus mit seinen Verhandlungszwängen zwischen den Ebenen genannt, sodass die Verantwortlichkeit für getroffene Entscheidungen unklar bleibt. Solche Verhandlungen finden sehr häufig informell und in Netzwerken statt. So wird auch im Konzept des ,network governance“ (Kohler-Koch/Eising 1999) die fehlende Verantwortlichkeit und die fehlende Input-Legitimation problematisiert (Papadopoulos 2003: 478; Sorensen 2002), insbesondere weil beteiligte Akteure nicht demokratisch legitimiert sein müssen. ${ }^{10}$ Jegliche Art der Informalisierung erhöht letztendlich den Bedarf an direktdemokratischer Beteiligung.

Aber auch eine Institutionalisierung und damit Formalisierung von Verhandlungen zwischen Staat und gesellschaftlichen Akteuren bringt demokratietheoretische Probleme mit sich (Czada 1995). In neo-korporatistischen Arrangements kann eine fehlende demokratische Input-Legitimierung der beteiligten gesellschaftlichen Akteure in Verbindung mit der Selektivität und Asymmetrie in der Beteiligung der Akteure zu Defiziten führen. Ersteres stellt infrage, ob die gesellschaftlichen Akteure tatsächlich die Betroffenen repräsentieren, und thematisiert die innerorganisatorische Demokratie von Verbänden (vgl. schon Michels 1911). Letzteres problematisiert, dass die Auswahl der gesellschaftlichen Akteure seitens des Staates selektiv ausfallen kann und unter Umständen eine ressourcenbasierte Asymmetrie vorherrscht (Abromeit/Stoiber 2006: 243). Als Antwort wird häufig das Argument eines dem Gemeinwohl förderlichen Outputs angeführt (Scharpf 1993). Doch können direktdemokratische Verfahren eine mögliche Output-Legitimation mit einer expliziten ex post erfolgten Input-Legitimierung versehen und so eine effektive Beteiligung ermöglichen.

10 Gegen diese Argumentation vgl. die Befürworter einer Effizienz- oder Output-Orientierung, z. B. Scharpf 1997; Schmitter 2000. 
Michael Stoiber

\section{Operationalisierung und Messung}

\section{a) Das optimale Mischverhältnis zwischen direkten und repräsentativen Instrumenten}

Das optimale Mischungsverhältnis hängt wie oben ausgeführt vom Grad der (In-)Formalisierung des Entscheidungssystems ab. Im Folgenden werden drei Erscheinungsformen vorgestellt, in denen sich (In-)Formalisierung zeigen kann:

1) Die Auswanderung und der Transfer der Entscheidungsfindung aus den formalen Institutionen in informelle Gremien und Treffen ist die erste Erscheinungsform. Es werden vier unterschiedliche Arten identifiziert, wie informelle Interaktion entstehen kann (von Blumenthal 2003): a) Konflikte innerhalb von Koalitionsregierungen werden in informellen Treffen zwischen den Parteispitzen und nicht innerhalb des Kabinetts gelöst; b) in bikameralen Systemen versuchen Parteivertreter die Überwindung von Blockadesituationen außerhalb der verfassungsgemäßen Prozeduren zu erreichen; c) Verhandlungen über Reformen finden zwischen Parteispitzen und nicht innerhalb der parlamentarischen Arena statt; d) die Politik-Formulierung wird von den Ministerien und parlamentarischen Ausschüssen in Experten-Kommissionen ausgelagert, wo u. a. Wissenschaftler und Interessenvertreter Programme ausarbeiten, die dann von den politischen Akteuren angenommen werden (zur EU Majone 1999). Ich schlage einen die vier Erscheinungsformen gleich zu gewichtenden additiven Index $I_{a}$ vor, der von 0 (keine dieser Auswanderungs-Arten) bis 1 (alle vier Arten) reichen kann.

2) Eine Einbindung privater Akteure existiert des Weiteren in einer recht neuen Art der Entscheidungsfindung, der sogenannten network governance. Das Ausmaß an network governance wurde bislang nicht systematisch in komparativer Perspektive analysiert, da Fallstudien die Forschung dominieren. Was daher zur Bildung eines Index verwendet werden kann, ist die Einschätzung, wie wichtig dieser neue Typ der Entscheidungsfindung insgesamt innerhalb eines politischen Systems ist. Dieser Index $I_{n}-$ der ebenfalls Werte zwischen 0 und 1 einnehmen kann - soll daher nur eine vorläufige subjektive Einstufung sein, die aufgrund der existierenden Literatur (z. B. Papadopoulos 2004; Savitch 1998) vorgenommen wird.

3) Neo-korporatistische Arrangements ermöglichen es Verbänden auf nationaler Ebene, den Entscheidungsprozess zu beeinflussen. Dieser Typ geht mit mehr oder weniger institutionalisierten Gremien einher, in denen private und öffentliche Akteure verhandeln und über (häufig ökonomische) Politiken entscheiden. Zur Messung des Korporatismusgrads wurden unterschiedliche Indikatoren entwickelt. Als etabliert kann inzwischen die Annäherung von Siaroff (1999) gelten. Er ordnet 24 OECD-Staaten anhand des Ausmaßes ihrer ,integrated economy“, das heißt einer koordinierten Steuerung der Wirtschaft unter Einbindung der Sozialpartner, ein. Für die Erstellung des Index $\mathrm{I}_{\mathrm{k}}$ wird seine Skala, die von 1 (kein Korporatismus) bis 5 (maximale Integration) reicht, entsprechend der Logik meines Modells auf den Werteraum 0 bis 1 normiert. 
Der Index des (In-)Formalisierungsgrades IF setzt sich aus den drei unterschiedlichen Typen zusammen, die jeweils gleich gewichtet Eingang finden.

(2) $\mathrm{IF}=\left(\mathrm{I}_{\mathrm{a}}+\mathrm{I}_{\mathrm{k}}+\mathrm{I}_{\mathrm{n}}\right) / 3$

Der optimale Anteil an direktdemokratischen Elementen bestimmt sich wie folgt:

(3) $\mathrm{k}=\frac{\mathrm{IF}}{2}$, mit $0 \leq \mathrm{IF} \leq 1$

Mit dieser Operationalisierung wird der maximale Wert von $\mathrm{k}$ auf 0,5 limitiert. Obwohl das als eine Ad-hoc-Festlegung erscheint, ist dieser Wert doch auch theoretisch plausibel, da ein System, das allein auf direkter Partizipation basiert, in modernen Nationalstaaten nicht machbar ist (Fuchs 2004: 99). Ohne indirekte Instrumente kann ein demokratisches System nicht funktionieren, weshalb ein System mit gleichen Anteilen an direkten und indirekten Instrumenten als das maximale Ausmaß direkter Demokratie gelten kann.

\section{b) Das optimale Angebot an Opportunitätsstrukturen}

Als baseline-model zur Identifikation einer optimalen Beteilungsstruktur wird das Konzept einer (minimalen) repräsentativen Demokratie für jene Länder gewählt, die sich einem gänzlich „unproblematischen“ Kontext gegenüber sehen, d. h. eine äußerst homogene Gesellschaft und ein einfaches Entscheidungssystem gehen ohne jegliche Informalisierung einher. In diesem Fall benötigen wir keinerlei direktdemokratische Instrumente, keine qualifizierten Mehrheiten oder Partizipationsmöglichkeiten auf verschiedenen Ebenen.

1) Gesellschaftliche Heterogenität jedoch ändert diese ideale Welt, minimale demokratische Institutionen auf Basis der einfachen Mehrheitsregel reichen nicht mehr aus (Lane 1996: 245). Unterschieden wird im Folgenden zwischen Erfordernissen aus expliziten Minderheitenrechten und solchen für segmentierte Gesellschaften. Für Letztere erscheint der Cleavage-Ansatz (Lipset/Rokkan 1967) für die Operationalisierung als nützlich. So identifiziert Lijphart (1999: 78 ff.) auf dieser Basis seine issue-dimensions, die von mittlerer oder hoher Bedeutung innerhalb des jeweiligen Systems sind. In segmentierten Gesellschaften ist die Gewährleistung von Repräsentation die erste institutionelle Herausforderung, die mittels eines proportionales Wahlsystem zu erreichen ist. Aber Repräsentation allein sichert keine effektive Beteiligung. Qualifizierte Mehrheiten müssen vorhanden sein, um einen größeren gesellschaftlichen Konsens zu gewährleisten und System-Instabilität zu vermeiden (Lijphart 1999: 32). 
Ich schlage daher vor, dass in Ländern mit maximal zwei issue-dimensions (nach Lijpharts Index [Lijphart 1999: 80]) die Anwendung der Mehrheitsregel ausreicht. ${ }^{11}$ Existiert eine geschriebene Verfassung, stellt diese den gesellschaftlichen Konsens über das Funktionieren des gemeinsamen Staatswesens dar, weshalb für Änderungen eine Supermajorität notwendig sein sollte. In der Realität erweist sich das formale Kriterium von 3/5-Mehrheiten als die niedrigste übliche parlamentarische Hürde, wie sie z. B. in Frankreich vorgesehen ist. ${ }^{12} \mathrm{Im}$ Falle eines Wertes von drei und mehr auf Lijpharts Index der issue-dimensions sollten qualifizierte Mehrheiten auch bei einfacher Gesetzgebung erreicht werden, da ansonsten das Risiko besteht, dass relevante Teile der Gesellschaft dauerhaft überstimmt werden. Das kann entweder durch formale qualifizierte Abstimmungsregeln oder durch übergroße Regierungskoalitionen erreicht werden. ${ }^{13}$ Solche Arrangements sind nicht notwendig, wenn es sich wie z. B. in Belgien oder der Schweiz bei der dritten issue-dimension um ethnisch-kulturelle Konflikte handelt, da hier die effektive Beteiligung mittels besonderer Minderheitenrechte umgesetzt werden kann.

Im Fall von Minderheiten kommen zwei unterschiedliche Mechanismen infrage, die eine effektive Selbstbestimmung gewährleisten können: Autonomierechte und Vetorechte (Goodin 1996: 639). Bei der Auswahl, welche Form die passende ist und den Bedarf am besten decken kann, ist auf die Stärke und insbesondere die regionale Konzentration der Minderheiten zu achten. Sind Minderheiten regional konzentriert und stellen dort die Mehrheit - wie im „klassischen“ Fall nationaler Minderheiten - kann die Gewährung von Autonomie- und damit Selbstregierungsrechten gewählt werden (Kymlicka 1995: 28). Dagegen können für nicht konzentrierte Minderheiten Vetorechte in jenen Bereichen installiert werden, die für eine eigenständige Identität grundlegend sind (vgl. für die EU Abromeit 1998: 103 ff.). Sind weder Autonomie- noch Vetorechte eingerichtet, kann eine Repräsentation der Minderheiten auf nationaler Ebene erfolgen. Das kann durch ein proportionales Wahlsystem mit möglichst niedrigen Repräsentationsschwellen oder eine garantierte Minderheitenvertretung erreicht werden. ${ }^{14}$ Doch ermöglicht Repräsentation zwar die Beteiligung am politischen Prozess, garantiert aber keine effektive Berücksichtigung der Präferenzen. Daher ist klar, dass die Mitbestimmung von Minderheiten allein auf Basis von Repräsentation in ihrem Effektivitätsmaß eingeschränkt bleibt.

11 Ich halte mich hier an Lijpharts Operationalisierung (Lijphart 1999: 78 ff.), in der er sieben Dimensionen mit längerfristiger Bedeutung identifiziert, die entweder stark (Wert 1) oder mittel (Wert 0,5) ausgeprägt sein können. Es wird davon ausgegangen, dass diese Dimensionen, die zur Herausbildung von Parteien führten, addiert werden können und mögliche Effekte eventuell überlappender Konflikte, die die Heterogenität abmildern, diese Logik nicht beeinträchtigen.

12 Eine Kombination von parlamentarischem Votum mit absoluter Mehrheit und Volksabstimmung wie in Dänemark kann auch als supermajoritär eingestuft werden (Lijphart 1999: 220).

13 Eine formale 2/3-Mehrheit bestand z. B. in Finnland bis 1992 für Steuergesetze (Abromeit/Stoiber 2006: 125), dagegen wurde in den Niederlanden jahrzehntelang der informelle Weg großer Koalitionen gegangen.

14 So z. B. in Neuseeland, wo zu Zeiten der Mehrheitswahl die Maori über vier eigene Wahlkreise verfügten. 
2) Bei einer hohen Komplexität bedarf es der Partizipation auf allen Ebenen. ${ }^{15}$ Zunächst macht eine Mehrebenenstruktur das institutionelle Arrangement komplexer (vertikale Komplexität). Dezentralisierung und Föderalismus sind zwei Wege, um die Staatsgewalt auf verschiedene Ebenen zu verteilen. Nach den unitarischen Systemen haben dezentrale die niedrigste Komplexität, da nur ein sehr beschränkter Anteil an Entscheidungskompetenzen von der nationalen Ebene nach unten delegiert ist. Es folgen die föderalen Systeme, bei denen die Komplexität im Fall des Trennföderalismus niedriger ausfällt als im Verbundföderalismus. Auf allen Entscheidungsebenen ist zu überprüfen, ob es Partizipationsmöglichkeiten gibt.

Auch auf horizontaler Ebene finden wir einen variierenden Komplexitätsgrad. Ein System wird komplexer, je mehr institutionelle Akteure als Vetospieler auftreten können (Tsebelis 2002; Stoiber 2007). In einem minimalen setting hätten wir allein ein unikamerales Parlament, doch können Zweite Kammern, Präsidenten und Verfassungsgerichte hinzukommen. ${ }^{16}$ Für Parlamentskammern und Präsidenten ist eine demokratische Legitimation notwendig; im Falle von Verfassungsgerichten ist zu prüfen, inwieweit die Bestellung der Richter durch demokratisch legitimierte Akteure erfolgt.

3) (In-)Formalisierung macht direktdemokratische Elemente genau auf der Ebene notwendig, auf der die informellen Entscheidungsstrukturen angesiedelt sind. Im Falle von Auswanderung aus den Institutionen und korporatistischen Arrangements sind daher Referenden auf nationaler Ebene notwendig. Dagegen ist network governance vor allem ein regionales oder lokales Phänomen, sodass die üblichen öffentlich-privaten Partnerschaften mittels direkter Beteiligungsmöglichkeiten kontrolliert werden müssen.

Am Ende ist der Bedarf an Opportunitätsstrukturen ( oso $_{\mathrm{r}}$ bzw. oso $_{\mathrm{d}}$ ) eine Zusammensetzung der soeben definierten Attribute und muss folglich mit dem tatsächlichen Angebot konfrontiert werden. Jeder identifizierten notwendigen institutionalisierten Beteiligungsmöglichkeit ( oso $_{\mathrm{r}}$ bzw. oso $_{\mathrm{d}}$ ) wird dann auf Angebotsseite ein osa bzw. osa $_{\mathrm{d}}$ mit dem Wert 1 (existiert) oder $0^{\mathrm{r}}$ (existiert nicht) zugeordnet. Die Gewichtung der einzelnen Elemente $\left(\alpha_{\mathrm{r}}\right.$ bzw. $\left.\alpha_{d}\right)^{17}$ hat kontextabhängig zu geschehen. Dazu ist jedoch die genaue Kenntnis des jeweiligen Kontextes (Gesellschaftsstruktur und Entscheidungssystem) notwendig, eine Festlegung der jeweiligen Bedeutung einzelner Elemente $\left(\alpha_{r}\right.$ bzw. $\left.\alpha_{d}\right)$ kann nicht a priori erfolgen. In ersten Anwendungen des Index muss daher zunächst von einer Gleichgewichtung aller Elemente ausgegangen werden. Zukünftig muss dann eine gesonderte Diskussion geführt werden, indem die Auswirkung unterschiedlicher induktiv abgeleiteter Gewichtungen auf den Gesamtwert DI fallweise überprüft wird.

15 Abromeit (2004: 85) berücksichtigt ebenfalls sektoral differenzierte Entscheidungszusammenhänge. Diese Ausdifferenzierung der Politik erscheint mir jedoch als zu kleinteilig, um sie für ein nationales Gesamtmaß an demokratischer Qualität berücksichtigen zu können. Teilweise findet sie sich im (In-)Formalisierungsgrad wieder (Auslagerung von Entscheidungen, korporatistische Arrangements).

16 Selbstverständlich kann das Volk selbst Vetospieler sein, die Frage nach einer demokratischen Legitimation stellt sich hier jedoch nicht, vielmehr später die Frage nach der Effektivität der Beteiligung.

17 Die von Munck/Verkuilen (2002: 22 ff.) sogenannte ,challenge of aggregation“. 


\section{c) Die Effektivität der bestehenden Opportunitätsstrukturen}

Doch das bloße Vorhandensein einer dem Bedarf entsprechenden Beteiligungsstruktur reicht nicht aus, um effektive Beteiligung und demokratische Qualität zu gewährleisten. Es ist ebenfalls zu prüfen, ob diese Strukturen auch effektiv genutzt werden können. Die Messung der Effektivität hat zwei Komponenten zu berücksichtigen: nicht allein die Wirksamkeit der bestehenden Strukturen (w), sondern auch das Ausmaß der Dominanzstrukturen (D). Ich schlage daher vor, die Wirksamkeit mit einem Dominanz-Indikator zu gewichten, da Dominanzstrukturen die Wirksamkeit aller demokratischen Instrumente reduzieren. Somit wird die Effektivität e folgendermaßen berechnet:

$$
\text { (4) } \mathrm{e}=\mathrm{w} \cdot(1-\mathrm{D})
$$

Durch die Multiplikation wird die Effektivität e null, wenn wir Strukturen finden, in denen - wie z. B. in totalitären Systemen - sämtliche Willensbildung top-down dominiert wird $(\mathrm{D}=1)$. Folglich wird auch der Gesamtindex DI null. Das ist ein plausibler Mechanismus, da das formale Vorhandensein von Opportunitätsstrukturen wie Wahlen und verantwortlicher Regierung im Falle eines dominanten Einparteiensystems nichts wert sind (Sartori 1976).

1) Zunächst widme ich mich der Messung der Dominanz D, da sie zur Bestimmung der Effektivität jeder Beteiligungsart notwendig ist. Ich verwende Katz' (1987) Konzept der partyness of society, um das Ausmaß an Dominanzstrukturen zu bestimmen (Abromeit/ Stoiber 2006). Im Falle einer hohen partyness of society ist es den Parteien möglich, ihre Ideologie und Vorstellungen in die Gesellschaft zu transportieren und so die Präferenzen der Bürger zu beeinflussen. Für Katz (1987: 8) fußt die partyness of society auf einer starken Verknüpfung zwischen Parteien und großen gesellschaftlichen Akteuren (Interessengruppen, Gewerkschaften), im folgenden $\mathrm{D}_{\mathrm{V}}$. Als zweites Element wird eine Versäulung oder Lagerbildung in Form von starken politischen Subkulturen herangezogen, die mit engen Beziehungen zwischen Partei-Eliten und der dazugehörigen Säule/Gruppe einhergeht $\left(D_{S}\right)$. Zusätzlich integriere ich das Mediensystem: Existieren politisierte Medien, wird die Verbindung zwischen Parteien und Öffentlichkeit enger und die Parteien können so die Präferenzen der Bürger stärker beeinflussen. Im Fall eines zusätzlich hoch konzentrierten oder monopolisierten Mediensystems ist das Ausmaß an Dominanz dann noch höher $\left(\mathrm{D}_{\mathrm{M}}\right)$. Als Indikatoren schlage ich einen Index vor, der für alle drei Elemente die Systeme gemäß den folgenden Kategorien einteilt:

\begin{tabular}{|l|l|}
\hline & Ausmaß an Dominanz (gilt für alle drei Elemente $\mathbf{D}_{\mathbf{v}}, \mathbf{D}_{\mathbf{S}}$ und $\mathbf{D}_{\mathbf{M}}$ ) \\
\hline 0 & Nicht vorhanden/sehr gering ausgeprägt \\
\hline 0,25 & Gering ausgeprägt \\
\hline 0,5 & Mittel \\
\hline 0,75 & Stark ausgeprägt \\
\hline 1 & Sehr stark ausgeprägt \\
\hline
\end{tabular}


Diese Einstufung muss in der Anwendung auf Basis der bestehenden Literatur und des Wissens über die jeweiligen Länder erfolgen und ist gegebenenfalls qualitativ zu begründen. Die identifizierten Elemente erscheinen in der Realität als von ähnlicher Bedeutung und Qualität, sodass es zwischen den drei Elementen keinerlei Gewichtung bedarf. Da sie sich jedoch gegenseitig bedingen und ggf. verstärken, wird der Dominanzindikator D mittels Multiplikation der drei Elemente berechnet. Gibt es z. B. keinerlei partyness of society, führt auch eine Monopolisierung des Mediensystems zu keiner Dominanz des Systems.

(5) $\mathrm{D}=\mathrm{D}_{\mathrm{V}} \circ \mathrm{D}_{\mathrm{S}} \circ \mathrm{D}_{\mathrm{M}}$

2) Die Wirksamkeit direktdemokratischer Instrumente hängt von vier potenziellen Restriktionen $\mathrm{r}$ ab (Hug/Tsebelis 2002). Sie ist reduziert, wenn a) eine Entscheidung nicht bindend ist, b) ein Referendum nicht vorgeschrieben ist und allein politische Akteure es initiieren können, c) es hohe Hürden gibt, um ein Referendum zu initiieren oder hohe Beteiligungsquoren für die Gültigkeit des Referendums existieren, d) es Einschränkungen in Bezug auf die möglichen Politikinhalte gibt. Während die ersten beiden Restriktionen $r_{1}$ und $r_{2}$ in Form eines dummies (trifft zu oder nicht) operationalisiert werden, können die Restriktionen drei und vier $\left(r_{3}\right.$ und $r_{4}$ ) mittels Indikatoren mit abgestuften Werten zwischen 0 und 1 nach dem Ausmaß der Einschränkung erfasst werden. Darüber hinaus ist zu diskutieren, ob die Häufigkeit der Nutzung des Instruments ebenfalls als Teil der Effektivität zu betrachten ist. Dagegen spricht jedoch, dass die identifizierten Restriktionen die Häufigkeit der Anwendung stark beeinflussen. Zudem spricht ein antizipativer Effekt des politischen Systems dagegen: Um Referenden mit eventuell nicht intendierten Ergebnissen zu verhindern, wird die politische Klasse solche Politiklösungen vorschlagen, die möglichst wenig Anlass zur Initiierung solcher Abstimmungen bieten.

Offen bleibt zu diesem Zeitpunkt noch eine Gewichtung der identifizierten vier Restriktionen, um den Parameter $\mathrm{w}_{\mathrm{D}}$ bestimmen zu können. Bis theoretische Argumente vorliegen, die eine Hierarchisierung oder eine Gewichtung begründen könnten, ist von einer Gleichgewichtung auszugehen, sodass sich die Wirksamkeit $\mathrm{w}_{\mathrm{D}}$ von direktdemokratischen Elementen bestimmt als:

(6) $\mathrm{W}_{\mathrm{D}}=1-\frac{\sum_{i=1}^{4} \mathrm{r}_{i}}{4}$

3) In Bezug auf die repräsentativen Instrumente ist die wichtigste Komponente, wie bedeutsam („meaningful“) die jeweiligen Wahlen sind. Nach Dahl (1971) liegen dann meaningful elections vor, wenn alle Stimmen gleich zählen und die Wahl einen Einfluss darauf hat, wer regiert. Diese Definition hilft aber lediglich, Demokratien von Diktaturen zu unterscheiden. Um Defizite innerhalb von Demokratien zu bestimmen, muss das Konzept jedoch erweitert werden. Aus Sicht der Individuen muss die Regierungsbildung nach den Wahlen mit klaren Policy-Implikationen verbunden sein, um ihre Wahlentscheidung gemäß ihren individuellen Erwartungen treffen zu können. Eingeschränkt ist dieses Prinzip, wenn a) ungeachtet des Wahlergebnisses immer die gleiche Partei den Kern der Regierung stellt, 
b) jegliche Koalition möglich erscheint und keinerlei Koalitionsaussagen vor der Wahl getroffen werden. ${ }^{18}$ Im Folgenden schlage ich einen Index vor, der zweistufig vorgeht: Handelt es sich um meaningful elections im Sinne von Dahl, wird $\mathrm{w}_{\mathrm{E}}$ der Wert 0,75 zugewiesen $^{19}$, je nach Anzahl der Einschränkungen des responsible party government steigt der Wert von $\mathrm{w}_{\mathrm{E}}$ bis auf 1,0 (keinerlei Einschränkungen). ${ }^{20}$

\begin{tabular}{|l|l|}
\hline $\mathbf{w}_{\mathbf{E}}$ & Wirksamkeit von Wahlen \\
\hline 0 & Keine freie Wahlen \\
\hline 0,75 & Mindestens, wenn bedeutsam gemäß Dahl \\
\hline 0,75 & Bei zwei Einschränkungen \\
\hline 0,875 & Bei einer Einschränkung \\
\hline 1 & Wenn keine Einschränkung vorliegt \\
\hline
\end{tabular}

4) Aber auch indirekte Wahlverfahren können die Effektivität reduzieren, da dadurch die Präferenzen der Bürger verzerrt wiedergegeben werden können. Der Verlust an Effektivität geht mit dem Ausmaß der Verzerrung einher, es ist auch hier ein $\mathrm{w}_{\mathrm{V}}$ zu bestimmen, das sich zwischen 1 (keine Verzerrungseffekte) und 0 (absolute Verzerrung) bewegt. Als Indikator kann hierfür die Disproportionalität zwischen der ursprünglichen Präferenzverteilung und der Mandatsverteilung in Form des Gallagher-Index verwendet werden (Lijphart 1999: 158), der auf den Werteraum von 0 bis 1 zu normieren ist.

5) Die Wirksamkeit der Repräsentation gesellschaftlicher Gruppen in segmentierten Gesellschaften $\mathrm{w}_{\mathrm{SG}}$ kann analog über den Gallagher-Index operationalisiert werden. Denn nur eine perfekte Proportionalität kann gewährleisten, dass die gesellschaftlichen Segmente gemäß ihrer politischen Präferenzen im Parlament vertreten sind.

6) Repräsentation reicht als alleiniges Instrument zur Sicherung von effektiver Minderheitenbeteiligung nicht aus, weshalb die Wirksamkeit $\mathrm{w}_{M}$ maximal den Wert 0,5 annehmen kann, wenn keine Autonomie- oder Vetorechte installiert sind. Der Wert selbst scheint zunächst relativ einfach mittels des Grades der Unterrepräsentierung in der zu wählenden Versammlung bestimmt werden zu können. Dazu ist für jedes Land zunächst zu definieren, welche Minderheiten als so relevant anzusehen sind, dass sie einer eigenen Repräsentation bedürfen. Die Wirksamkeit $\mathrm{w}_{\mathrm{M}}$ bestimmt sich dann mittels des Anteils an Mandaten, die die

18 Beide Elemente können auf die Idee des ,responsible party government“ (vgl. schon Ranney 1954) zurückgeführt werden. Die dort zusätzliche Implikation, dass elektorale Verluste sich in der Beendigung der Regierungsbeteiligung niederschlagen sollen, ist dagegen aus der eingenommenen individualistischen Sichtweise nicht zwingend.

19 Diese Ad-hoc-Festlegung fußt auf der Überlegung, dass Mängel in der demokratischen Qualität bei Erfüllung von Dahls Kriterium den Wert von Wahlen nur bedingt einschränken können, ein Wert von 0,5 daher auf jeden Fall eine zu starke Zurückstufung bedeuten würde.

20 Defekten Demokratien oder Nicht-Demokratien sind dann Werte zwischen 0 und 0,75 zuzuordnen. Eine genaue Operationalisierung bleibt an dieser Stelle aufgrund des Fokus auf etablierte westliche Demokratien aus. 
jeweiligen Minderheitenvertreter innehaben, im Verhältnis zum Bevölkerungsanteil der Minderheit. Bei dieser Operationalisierung wird dann davon ausgegangen, dass die jeweilige Minderheit auch ,ihre“ Vertreter wählt. Was ist jedoch in den Fällen, in denen die Mitglieder der Minderheit sich bewusst für andere Parteien entscheiden? ${ }^{21}$ In einer konservativen Operationalisierung muss daher wieder auf die Existenz der Möglichkeit der Minderheitenrepräsentation zurückgegriffen werden, d. h. existiert ein dafür geeignetes Wahlsystem. In diesem Fall ist der Wert von $\mathrm{w}_{\mathrm{M}}$ gleich 0,5 bei rechtlich garantierten Mandaten für Minderheiten bzw. bestimmt sich wieder über den Gallagher-Index, der dann auf den Wertebereich von 0 bis 0,5 zu normieren ist. Es sollten in den ersten empirischen Anwendungen beide Alternativen getestet werden, inwieweit sie zu unterschiedlichen Ergebnissen führen und welche Auswirkungen sie auf den Gesamtindex haben, bevor eine endgültige Festlegung erfolgt.

Schwieriger gestaltet sich die Beurteilung der Effektivität von Veto- und Autonomierechten. Für jedes Land sind zunächst jene Politikfelder zu identifizieren, die grundlegend für die Identität der jeweiligen Minorität sind. Liegt in diesen Feldern keine Autonomie vor und existieren keine Vetorechte, kann das als Defizit identifiziert werden. Die Wirksamkeit $\mathrm{W}_{\mathrm{AV}}$ bestimmt sich dann als der Anteil der Politikbereiche, für die Autonomie- oder Vetorechte gewährleistet sind. Das grundlegende Problem dieses Aspekts der Demokratiemessung ist die Bestimmung des Sollzustandes, also jener Politikfelder, die mit besonderen Rechten versehen sein müssten. Ab wann impliziert eine eigene kulturelle Identität z. B. die Möglichkeit autonomer Regelungen im Bildungssystem? Die Forderungen seitens der Akteure selbst scheinen ein unzureichender Indikator zu sein, Kriterien für eine objektive externe Beurteilung sind schwierig (Pan/Pfeil 2002). Folglich können in einer ersten Anwendung allein offensichtliche Defizite identifiziert werden.

\section{Fazit}

Mit dem vorgestellten Modell eines kontextualisierten Maßes wird der Versuch unternommen, auf Basis eines nahezu minimalen Demokratieverständnisses Varianz in der demokratischen Qualität von Demokratien finden zu können. Damit wird ein alternativer Weg zu solchen Vorhaben eingeschlagen, die dieses Ziel durch eine umfassendere Demokratiekonzeption erreichen wollen (Merkel 1999; Bühlmann u. a. 2006, 2007). Das Auffinden von Varianz wird möglich, indem aus den gesellschaftlichen und institutionellen Kontextbedingungen ein jeweils individueller Bedarf an optimalen Beteiligungsstrukturen abgeleitet werden kann. Erst eine solche kontextspezifische Vorgehensweise kann eine Überprüfung der in der Demokratiedefinition eingeforderten effektiven Partizipation der Individuen an den kollektiven Entscheidungsprozessen ermöglichen. Denn durch die Konfrontation mit den tatsächlich gegebenen Strukturen und deren Effektivität kann dann die Identifikation länderspezifischer Defizite erfolgen.

Somit eignet sich das vorgestellte Modell, um Defizite in der demokratischen Qualität für jedes einzelne Land qualitativ zu entdecken. Mittels des Demokratie-Index DI soll es aber auch möglich werden, das Ausmaß an Defiziten zu quantifizieren und über Länder hinweg

21 Diese Problematik ist analog zur Frage, inwiefern die Wahlbeteiligung ein Kriterium zur Bestimmung demokratischer Qualität sein kann. 
vergleichbar zu machen. Die aus den Kontextbedingungen abgeleitete optimale Verteilung von repräsentativen und direkt-demokratischen Elementen $(\mathrm{k})$ stellt eine erste länderspezifische Gewichtung dar. Ein Weniger bei direktdemokratischen Partizipationsmöglichkeiten in Land A gegenüber Land B bedeutet noch nicht automatisch eine geringere Demokratiequalität, da gegebenenfalls der Bedarf an direkter Partizipation in Land A niedriger als in Land B ausfällt. Innerhalb der repräsentativen und der direktdemokratischen Elemente wird eine additive Logik mit einer Gewichtung der einzelnen institutionalisierten Mechanismen als Aggregationsregel angewandt, um jeweils spezifische gesellschaftliche und institutionelle Rahmenbedingungen berücksichtigen zu können. Hierin liegt aber auch noch ein Problem für die empirische Anwendung des Demokratie-Index DI. Die kontextabhängige Gewichtung $\alpha$ der einzelnen Opportunitätsstrukturen ist zwar theoretisch aus der Logik des kontextualisierten Modells heraus notwendig, aber schwer deduktiv abzuleiten. Hier scheint ein induktives Vorgehen notwendig, das erst in der empirischen Anwendung und unter Kenntnis der jeweiligen Länder eine begründete Festlegung der jeweiligen $\alpha$ ermöglicht.

Für die Umsetzung der Messung stellt sich noch ein weiteres Problem, das schon aus der klassischen Demokratiemessung bekannt ist. Der erste Schritt ist eine gute Begründung in der Auswahl der Indikatoren auf Basis des gewählten Konzepts (Munck/Verkuilen 2002). Im vorliegenden Fall ergibt sich die Auswahl direkt aus der Logik des Modells, für viele Indikatoren stellt sich unter methodischen Gesichtspunkten aber das Problem der Subjektivität bei der Wertevergabe für die jeweiligen Länder. Nur wenige Indikatoren verfügen über eine externe Validierung, wie z. B. der Korporatismus-Grad nach Siaroff (1999) oder der GallagherIndex zur Bestimmung von Disproportionalität. Für alle anderen Indikatoren gilt, dass die Übersetzung des qualitativen Wissens um die einzelnen Politikprozesse in einen konkreten Wert einer transparenten Begründung im Einzelfall bedarf. Als eine übliche Methode der externen Validierung sowohl der einzelnen Werte als auch der Indikatorwahl selbst kann hier mit Experteneinschätzungen gearbeitet werden, wie sie z. B. auch für den neuen BertelsmannReform-Index angewendet werden (Brusis 2008).

So zeichnet sich ab, dass eine erste empirische Anwendung des kontextualisierten Modells zunächst mit einem kleinen $\mathrm{n}$ zu erfolgen hat, um das notwendige Wissen über die einzelnen Systeme adäquat einzusetzen und die Werte der einzelnen Indikatoren gut begründen zu können. Ein Vergleich sollte dann jedoch sowohl qualitativ als auch quantitativ möglich sein.

\section{Literatur}

Abromeit, Heidrun, 1998: Democracy in Europe. Legitimising Politics in a Non-State Polity, New York/Oxford.

Abromeit, Heidrun, 2002: Wozu braucht man Demokratie? Die postnationale Herausforderung der Demokratietheorie, Opladen.

Abromeit, Heidrun, 2004: Die Messbarkeit von Demokratie: Zur Relevanz des Kontexts, in: PVS 45, 73-93.

Abromeit, Heidrun/Stoiber, Michael, 2006: Demokratien im Vergleich. Einführung in den Vergleich politischer Systeme, Wiesbaden. 
Beetham, David (Hrsg.), 1994: Defining and Measuring Democracy, London.

Berlin, Isaiah, 1995: Freiheit. Vier Versuche, Frankfurt a. M.

Blumenthal, Julia von, 2003: Auswanderung aus den Verfassungsinstitutionen, in: Aus Politik und Zeitgeschichte, B 43, 9-15.

Böckenförde, Ernst W., 1991: Staat, Verfassung, Demokratie. Studien zur Verfassungstheorie und zum Verfassungsrecht, Frankfurt a. M.

Brusis, Martin, 2008: Reformfähigkeit messen? Konzeptionelle Überlegungen zu einem Reformfähigkeitsindex für OECD-Staaten, in : PVS 49, 92-113.

Bühlmann, Marc/Hardmeier, Sibylle/Merkel, Wolfgang/Müller, Lisa/Wessels, Bernhard, 2006: Quality of Democracy. Democracy Barometer for Established Democracies: Konzeptualisierung eines neuen Instruments zur Messung von Demokratiequalität, NCCRDemocracy, Universität Zürich/WZB.

Bühlmann, Marc/Merkel, Wolfgang/Wessels, Bernhard, 2007: The Quality of Democracy: Democracy Barometer for Established Democracies, NCCR-Democracy Working Paper 10, Universität Zürich/WZB.

Croissant, Auriel/Thiery, Peter, 2000: Defekte Demokratie. Konzept, Operationalisierung und Messung, in: Hans-Joachim Lauth/Gert Pickel/Christian Welzel (Hrsg), Demokratiemessung, Wiesbaden, 89-111.

Czada, Roland, 1995: Konjunkturen des Korporatismus. Zur Geschichte eines Paradigmenwechsels in der Verbändeforschung, in: Wolfgang Streeck (Hrsg.), Staat und Verbände, Opladen (PVS-Sonderheft 25), 37-64.

Dahl, Robert A., 1971: Polyarchy. Participation and Opposition, New Haven/London.

Dahl, Robert A., 2000: On Democracy, New Haven.

Diamond, Larry/Morlino, Leonardo, 2004: The Quality of Democracy. An Overview, in: Journal of Democracy 15 (4), 14-25.

Fuchs, Dieter, 2004: Konzept und Messung von Demokratie. Eine Replik auf Heidrun Abromeit, in: PVS 45, 94-106.

Goodin, Robert E., 1996: Designing Constitutions: The Political Constitution of a Mixed Commonwealth, in: Political Studies 44 (3), 635-646.

Hug, Simon/Tsebelis, George, 2002: Veto Players and Referendums around the World, in: Journal of Theoretical Politics 14 (4), 465-515.

Katz, Richard S., 1987: Party Government and its Alternatives, in: ders. (Hrsg.), Party Governments: European and American Experiences, Berlin/New York, 1-26.

Kohler-Koch, Beate/Eising, Rainer, 1999: The Evolution and Transformation of European Governance, in: Beate Kohler-Koch/Rainer Eising (Hrsg.), The Transformation of Governance in the European Union, London, 14-35.

Kymlicka, Will, 1995: Multicultural Citizenship. A Liberal Theory of Minority Rights, Oxford.

Lane, Jan-Erik, 1996: Constitutions and Political Theory, Manchester.

Lauth, Hans-Joachim, 2004: Demokratie und Demokratiemessung. Eine konzeptionelle Grundlegung für den interkulturellen Vergleich, Wiesbaden.

Lauth, Hans-Joachim, 2008: Die Qualität der Demokratie. Der NID als pragmatischer Vorschlag für die komparative Forschung, in: Joachim Behnke/Nathalie Behnke/Kai-Uwe Schnapp, Datenwelten. Datenerhebung und Datenbestände jenseits klassischer Umfrageformen, Baden-Baden i. E. 
Lauth, Hans-Joachim/Pickel, Gert/Welzel, Christian, 2000: Demokratiemessung. Konzepte und Befunde im internationalen Vergleich, Wiesbaden.

Lijphart, Arend, 1995: Self Determination versus Predetermination of Ethnic Minorities in Power-Sharing Systems, in: Will Kymlicka (Hrsg.), The Rights of Minority Cultures, Oxford, 275-287.

Lijphart, Arend, 1999: Patterns of Democracy. Government Forms and Performance in Thirty-Six Countries, New Haven.

Lipset, Seymour/Rokkan, Stein, 1967: Cleavage Structures, Party Systems, and Voter Alignments: An Introduction. in: dies. (Hrsg.), Party Systems and Voter Alignments: CrossNational Perspectives, New York, 1-64.

Majone, Giandomenico, 1999: The Regulatory State and Its Legitimacy Problems, in: West European Politics 22, 1-23.

Merkel, Wolfgang, 1999: Defekte Demokratien, in: ders./Andreas Busch (Hrsg.), Demokratie in Ost und West, Frankfurt a. M., 361-381.

Merkel, Wolfgang, 2003: „Eingebettete“ und defekte Demokratien: Theorie und Empirie, in: Claus Offe (Hrsg.), Demokratisierung der Demokratie. Diagnosen und Reformvorschläge, Frankfurt a. M./New York, 43-71.

Merkel, Wolfgang/Puhle, Hans-Jürgen/Croissant, Aurel/Eicher, Claudia/Thierry, Peter, 2003: Defekte Demokratie, Band 1: Theorien und Probleme, Wiesbaden.

Merkel, Wolfgang/Puhle, Hans-Jürgen/Croissant, Aurel/Eicher, Claudia/Thiery, Peter, 2006: Defekte Demokratie, Band 2: Regionalanalysen, Wiesbaden.

Meyer, Thomas, 2005: Theorie der Sozialen Demokratie, Wiesbaden.

Michels, Robert, 1911: Zur Soziologie des Parteiwesens in der modernen Demokratie. Untersuchungen über die oligarchischen Tendenzen des Gruppenlebens, Leipzig.

Munck, Gerardo L./Verkuilen, Jay, 2002: Conceptualizing and Measuring Democracy: Evaluating Alternative Indices, in: Comparative Political Studies 35 (1), 5-34.

Pan, Christoph/Pfeil, Beate S., 2002: Minderheitenrechte in Europa, Wien.

Papadopoulos, Yannis, 2003: Cooperative Forms of Governance: Problems of Democratic Accountability in Complex Environments, in: European Journal of Political Research 42, 473-501.

Papadopoulos, Yannis, 2004: Governance und Demokratie, in: Arthur Benz (Hrsg.), Governance - Regieren in komplexen Regelsystemen, Opladen, 215-237.

Ranney, Austin, 1954: The Doctrine of Responsible Party Government. Its Origins and Present State, Urbana.

Sartori, Giovanni, 1976: Parties and Party Systems, Cambridge.

Savitch, Hank V., 1998: The Ecology of Public-Private Partnerships: Europe, in: Jon Pierre (Hrsg.), Partnerships in Urban Governance, Houndmills, 175-186.

Scharpf, Fritz W., 1993: Versuch über Demokratie im verhandelnden Staat, in: Roland Czada/Manfred G. Schmidt (Hrsg.), Verhandlungsdemokratie, Interessenvermittlung, Regierbarkeit, Opladen, 25-50.

Scharpf, Fritz W., 1997: Economic Integration, Democracy and the Welfare State, in: Journal of European Public Policy 4, 18-36.

Schmidt, Manfred G., 2000: Demokratietheorien. Eine Einführung, Opladen (3. Aufl.). 
Schmitter, Phillippe C., 2000: How to Democratize the EU ... and why Bother?, Lanham. Siaroff, Alan, 1999: Corporatism in 24 Industrial Democracies: Meaning and Measurement, in: European Journal of Political Research 36 (2), 175-205.

Sorensen, Eva, 2002: Democratic Theory and Network Governance, in: Administrative Theory and Praxis 24 (4), 693-720.

Stoiber, Michael, 2007: Gewaltenteilung, Machtteilung und das Vetospieler-Konzept, in: ZPol 17, 21-41.

Taylor, Charles, 1993: Multikulturalismus und die Politik der Anerkennung, hrsg. von Amy Gutman, Frankfurt a. M.

Tsebelis, George, 2002: Veto Players. How Institutions Work, New York/Princeton.

Vanhanen, Tatu, 1997: Prospects of Democracy. A Study of 172 States, London.

Korrespondenzanschrift:

Dr. Michael Stoiber

Institut für Politikwissenschaft

TU Darmstadt

Residenzschloss

64283 Darmstadt

E-Mail: mstoiber@pg.tu-darmstadt.de

Web: http://www.politikwissenschaft.tu-darmstadt.de/index.php?id=408

Der Autor vertritt die Professur „Politisches System der BRD/Vergleich politischer Systeme“ am Institut für Politikwissenschaft der TU Darmstadt. 